\title{
Práticas lean nos processos produtivos industriais: ações para a redução de custos $e$ resíduos de matéria-prima
}

\section{Lean practices in industrial production processes: innovations that provide reduction in costs and raw materials residues}

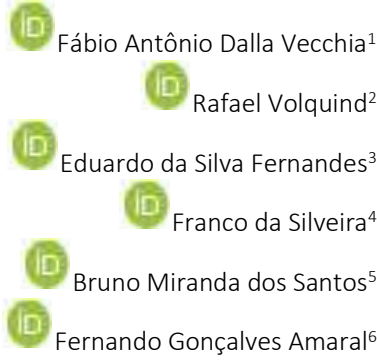

1Universidade Federal do Rio Grande do Sul. Engenheiro de Produção e Transportes pela Universidade Federal do Rio Grande do Sul (UFRGS). Mestre em Engenharia de Produção pelo Programa de Pós-Graduação em Engenharia de Produção da Universidade Federal do Rio Grande do Sul, na área de Sistemas de Produção. Membro do Núcleo de Capacitação em Ergonomia, Saúde e Segurança Ocupacional (NECSSO) do Laboratório de Otimização de Produtos e Processos (LOPP). fdvecchia@gmail.com

${ }^{2}$ Universidade Federal do Rio Grande do Sul. Engenheiro Civil pela Universidade Federal do Rio Grande do Sul. Mestre em Engenharia de Produção pelo Programa de Pós-Graduação em Engenharia de Produção da Universidade Federal do Rio Grande do Sul. MBA em Perícia, Auditoria e Gestão Ambiental pelo Instituto de Graduação e Pós-Graduação. Especialista em Saneamento e Engenharia Ambiental de Obras Civis pela Pontifícia Universidade Católica do Rio Grande do Sul. Chefe do Departamento de Gestão Descentralizada da Fundação Estadual de Proteção Ambiental (FEPAM/RS).rvolquind@gmail.com

Universidade Federal do Rio Grande do Sul. Doutorando e Mestre em Engenharia de Produção pela Universidade Federal do Rio Grande do Sul (UFRGS), graduado em Engenharia Elétrica pela Universidade do Estado de Santa Catarina (UDESC). Tem interesse pelas áreas de previsão de demanda, estatística, projeto de experimentos e Controle Estatístico do Processo (CEP). edu.silva.fernandes@gmail.com

${ }^{4}$ Universidade Federal do Rio Grande do Sul. Doutorando em Engenharia de Produção n Universidade Federal do Rio Grande do Sul (UFRGS/2018-2022). Membro do Núcleo de Capacitação em Ergonomia, Saúde e Segurança Ocupacional (NECSSO) do Laboratório de Otimização de Produtos e Processos (LOPP). Mestrado em Engenharia de Produção pela

Universidade Federal de Santa Maria (UFSM, 2018). franco.da.silveira@hotmail.com

5Universidade Federal do Rio Grande do Sul. Doutorando em Engenharia de Produção pela Universidade Federal do Rio Grande do Sul (UFRGS). Mestre em Engenharia de Produção pela Universidade Federal de Santa Maria (UFSM). Graduado em Engenharia de Produção pela UFSM. Pesquisador do Núcleo de Estratégias em Operações Produtivas (NEOP/20182019) do Instituto Federal de São Paulo (IFSP). Avaliador dos periódicos: Revista de Gestão Organizações Cooperativas (RGC)/2017-2018, Independent Journal Management \& Production (IJMP) 2018-2019 e Revista Exacta/2018-2019. Desenvolve pesquisas principalmente nas seguintes áreas: Gestão de operações em saúde, Leanhealthcare, Gestão da Cadeia de Suprimentos, Green Suplly Chain Management, Estratégias em produção e Teoria dos Conjuntos Fuzzy para tomada de decisões. brmiranda10@gmail.com

${ }^{6}$ Universidade Federal do Rio Grande do Sul. Fernando Gonçalves Amaral, engenheiro civil, especialista em ergonomia pela Université Catholique de Louvain (UCL), Bélgica (1991) mestre em ergonomia pela UCL (1992), mestre em ergonomia pela École Pratique de Hautes Études, Sorbonne, França (1995), doutor em ergonomia pela Université Catholique de Louvain (UCL), Bélgica em 1999. Atualmente é Professor Associado 4 da Universidade Federal do Rio Grande do Sul e orientador de mestrado e doutorado no Programa de Pósgraduação em Engenharia de Produção.amaral@producao.ufrgs.br

\section{Resumo}

O controle dos processos em empresas que estão em rápida expansão pode ser complexo e de alto custo se não for feito de forma organizada e planejada. A combinação da disposição dos processos, da alta variabilidade de peças produzidas e de um grande volume fabricado pode ser um fator que aumenta a propensão de perdas. Nesse contexto, este estudo tem como objetivo, com base nas práticas lean, propor melhorias no processo de corte de chapas finas de uma linha produtiva de uma fábrica de elevadores localizada na região sul do Brasil. Em termos metodológicos, foi selecionado o estudo de caso. A pesquisa classifica-se como descritiva, de caráter exploratório. Como resultados, obtevese reduções significativas das perdas ocorridas no processo atual, com ganhos em qualidade, redução de estoque, diminuição de retrabalhos e aumento de produtividade através da aplicação de técnicas e ferramentas lean.

Palavras-chave: Controle de processos. Produção Enxuta. Lean. Melhorias.

\section{Abstract}

Controlling processes in rapidly expanding companies can be complex and costly if not done in an organized and planned way. The combination of the arrangement of processes, the high variability of produced parts and a large volume manufactured can be a factor that increases the propensity of losses. In this context, this study aims, based on lean practices, to propose improvements in the thin sheet cutting process of a production line of an elevator plant located in the southern region of Brazil. In methodological terms, the case study was selected. The research is classified as descriptive, of an exploratory nature. As results, significant losses were achieved in the current process, with gains in quality, inventory reduction, reduction of rework and productivity increase through the application of lean tools and techniques.

Keywords: Process Control. Lean Production. Lean. Improvements. 


\section{Introdução}

O aumento da competitividade devido às condições atuais do mercado faz com que as empresas repensem a eficiência de seus processos produtivos (Utterback \& Abernathy, 1975; Lermen et al., 2018; Marodin et al., 2018; Pearce, Pons \& Neitzert, 2018). Dentro deste contexto, as empresas se vêm obrigadas a aprimorar seus processos produtivos, bem como as ferramentas utilizadas no controle da produção, buscando redução de perdas, sendo esta uma das principais razões para a adoção de práticas lean (Salgado, Mello, Silva, Oliveira \& Almeida, 2009; Palisaitiene, Sundin \& Poksinska, 2018). Organizações que atuam há muitos anos no mercado e obtiveram sucesso nesse período normalmente apresentam resistência à mudança, estagnando suas inovações. Tal estagnação é derivada de diversos fatores, com destaque para dois: primeiramente, por seu histórico de sucesso (é normal que não aconteça busca por melhorias quando os resultados são positivos), devido também ao histórico protecionista do país (Coronel, 2014; Negri \& Cavalcante, 2013); e segundo, por seus processos terem se tornado complexo devido ao crescimento desestruturado e existir uma resistência por parte das pessoas de aceitar a reestruturação dos processos (Shah \& Ward, 2003; Este, lammarino, Savona \& Tunzelmann, 2012; Bergek et al., 2015). A forma de operação e gerenciamento muitas vezes torna-se complicada, pois há uma inércia nos processos, que são verticalizados, com baixo nível de controle. Este cenário apesar de ser comum, dificulta os projetos de melhorias, os quais se tornam burocráticos e complexos (Cançado \& Santos, 2014; Alkhoraif \& McLaughlin, 2018).

Para lidar com estes desafios, as organizações têm adotado nas últimas décadas o Sistema de Produção Lean (LPS). Várias organizações que adotaram o LPS chegaram a bons resultados diretos esperados (Hasibul, Gustav \& Malin, 2018), além de outros, subjetivos, que também são observados, como aumento da satisfação com o trabalho, melhoria do ambiente organizacional e uma comunicação mais efetiva entre os colaboradores (Sharma \& Gandhi, 2017). Além disso, tem-se aumentado a exigência por parte dos consumidores a respeito da flexibilidade, variedade e velocidade de entrega dos produtos (Oliveira, 2009).

O objetivo deste estudo é avaliar os possíveis efeitos que a implementação de técnicas e ferramentas da produção enxuta podem trazer para um setor produtivo de uma fábrica de elevadores localizada na região sul do Brasil. Assim, foi necessário avaliar e descrever a situação anterior à intervenção, explicar as técnicas utilizadas e, posteriormente, os resultados obtidos e discussões posteriores. Os problemas abordados foram relacionados à perda por armazenamento, movimentação e fabricação de peças defeituosas.

Este trabalho está organizado da seguinte forma. Na seção 2, é contextualizado os conceitos que se relacionam com o lean production. A seção 3 apresenta a metodologia implementada e suas respectivas justificativas. Na seção 4 há a descrição da situação anterior e o detalhamento das condições 
assim como a identificação da possibilidade de ganhos do processo e subprocessos. A secção 5 são apresentados e discutidos os resultados obtidos em relação aos objetivos propostos. Finalmente, a seção 6 apresenta as considerações finais, limitações e propostas para trabalhos futuros.

\section{Referencial Teórico}

O Sistema Toyota de Produção, comumente conhecido como lean production, caracteriza-se como um conjunto de princípios, práticas e ferramentas usadas para criar valor ao consumidor, com melhor qualidade e poucos defeitos, com menos esforços humanos, menos utilização de espaço físico, menos capital e menos tempo do que os sistemas tradicionais de produção em massa (Liker, 2004; Corte et al., 2016). De forma mais objetiva, reduz continuamente as perdas em todas as áreas e de todas as formas (Bortolotti, Boscari, \& Danese, 2015; Soliman \& Saurin, 2017). Conforme Womack e Jones (2004), a base da produção enxuta consiste em eliminar os desperdícios para reduzir os custos de produção e maximizar o valor agregado.

A produção enxuta tem como fundamento eliminar, ou quando não for possível, mitigar as perdas ocorridas no processo de produção (Hines, Holweg, \& Rich, 2004; Fliedner \& Majeske, 2010; Ledón et al., 2018). Para Ohno (1988) e Shingo e Dillon (1989), as sete principais perdas são: por superprodução (causada pela produção excessiva ou antecipada, acarreta em estoque e é a mais danosa); por espera (quando um produto fica estacionado esperando a luz verde para ir para a próxima etapa); por transporte (ocupa em média metade do tempo total de fabricação); no processamento (etapas que podem ser eliminadas sem causar dano ao valor do produto); por estoque (tanto de matéria-prima como entre as etapas de produção, que ocasiona gastos com espaço físico e manutenção); por movimentação (movimentos desnecessários realizados pelos operadores); e por fabricação de produtos defeituosos (produtos que não satisfazem aos requisitos de uso).

Segundo Slack et al. (2002), para implementar o sistema Lean, é necessário utilizar técnicas ou ferramentas, tais como kanban, setup rápido, entre outras. Tratam-se de técnicas que procuram eliminar atividades que não agregam valor. O uso de tais ferramentas e estratégias auxiliam na simplificação de todos os aspectos dos processos por meio do ataque direto a cada tipo de perda originada pelos sistemas produtivos (Aherne \& Whelton, 2010). A gestão de estoques é considerado de suma importância neste processo, tendo relação direta com a qualidade e redução de custos (Oliveira, 2009).

Neste estudo, além da análise das sete perdas, foram executadas ações e aplicadas ferramentas como o kanban, sequenciamento de ordens de produção e organização de sobras de matéria-prima.O sistema kanban é um mecanismo que informa o momento para reabastecer ou produzir exatamente o 
que está sendo requerido (Womack \& Jones, 2004). Tornando o material necessário para tal produção sempre disponível no momento em que é requerido. Já tecnologia da informação é a utilização de sistemas informatizados para o apoio às operações (Giannini, 2007). Neste caso, dando ao operador as informações necessárias para saber o que deverá ser produzido e em ordem otimizada para reduzir os tempos de setup. O nivelamento da produção, que procura manter o volume total produzido o mais constante possível (Giannini, 2007), entre outras. Existe uma grande quantidade de ferramentas para práticas lean já desenvolvidas e à medida que se avança no desenvolvimento de conhecimentos, mais ferramentas surgem com o objetivo de reduzir as perdas e melhorar os processos produtivos.

\section{Procedimentos Metodológicos}

A pesquisa classifica-se quanto à sua abordagem como qualitativa. Do ponto de vista dos seus objetivos, a pesquisa caracteriza-se como descritiva, de caráter exploratório (Gil, 2012). Referente aos procedimentos técnicos utilizados, este trabalho pode ser considerado como um estudo de caso em uma indústria fabricante de elevadores localizada na região sul do Brasil (Yin, 2001).

O presente estudo foi realizado em uma empresa multinacional fabricante de elevadores. A fábrica onde foi realizado o estudo foi adquirida pela multinacional aproximadamente há duas décadas e apresenta histórico de administração familiar. É responsável por produzir elevadores comerciais, residenciais e de carga. Além disso, é uma das empresas líder no nicho de mercado que possui atuação. Seus produtos são caracterizados pela alta customização, o que torna seu processo produtivo custoso e complexo. Existem processos padrão, porém há muita variabilidade entre os itens que formam o produto final.

Com base no sistema de produção enxuta, fez-se uma análise das sete perdas de produção e, com base nos relatórios de perdas da empresa, focou-se nos três principais fatores que impactam na eficiência: estoque, movimentação e fabricação de produtos defeituosos. De modo que, foram aplicadas as técnicas oriundas do sistema de produção enxuta, como a implementação de sequenciamento para redução de quantidade e tempo de setups e Kankan, que proporciona a redução do tempo de espera e aumento da disponibilidade de material.

\subsection{Descrição do Cenário}

A produção da fábrica é dividida em duas grandes fases: i) transformação: corte, dobra, estamparia, soldagem, usinagem e pintura; e ii) montagem: os itens provenientes da transformação e externos à fábrica são pré-montados ou separados para serem enviados aos clientes. 
As Ordens de Fabricação (OF), listagens com os itens que devem ser fabricados ao longo do dia, são geradas pelo Departamento de Planejamento e Controle da Produção (DPCP) e recebidas pelos setores iniciais do processo. Como há uma grande variabilidade de itens produzidos e, devido à necessidade de aproveitar ao máximo a matéria-prima e a carga de máquina, as OFs são geradas com frequências diária, semanal e quinzenal, proporcionando a redução da quantidade de setups.

Os setores da transformação são divididos por função: corte de chapas finas (foco do estudo), corte de chapas grossas, usinagem, dobra, prensa, solda e pintura. A montagem por sua vez possui arranjo celular onde cada célula é responsável pela pré-montagem das partes-chave do elevador.

\subsection{Identificação das Perdas}

O setor de chapas finas contempla: sete funcionários, estoque de chapas, guilhotina, puncionadeira, dobradeira e robô de solda. É um processo crítico da fábrica devido à grande variabilidade e fragilidade da matéria-prima. Neste setor são produzidos painéis para a fabricação de portas com chapas de aço entre 0,9 e 1,2 milímetros de espessura. Os maiores problemas observados e levantados pelos colaboradores do setor se referem a excessiva troca de matéria-prima, dificuldade de reaproveitamento de material, grande quantidade de incidentes de urgência (produção de algum item que não estava na programação inicial). Estes fatores aumentam significativamente o tempo de setup e espera por empilhadeira para o transporte da MP. Além disso, a grande quantidade de MP em estoque também é apontada como um problema.

A guilhotina é o primeiro posto por onde passam as portas. Elas recebem as chapas de aço, que são cortadas conforme descrito na ordem de fabricação. No momento em que foi realizado o estudo, as guilhotinas não possuíam plano de corte, ficando a cargo dos operadores decidirem qual é a melhor forma de aproveitamento das chapas. Assim como o plano de corte, também não existem sequenciamento e controle de estoque. Estes fatores têm grande influência nos processos seguintes, que atingem diretamente os setores de dobra, colagem e montagem das portas, gerando movimentações excessivas, número elevado de Não Conformidades (NC) e sua identificação tardia. As NCs encontradas foram amassos, arranhões e produção excedente. Um esquema da metodologia deste estudo foi criado para facilitar o entendimento do desenvolvimento do trabalho. A Figura 1 apresenta o framework desenvolvido para esta pesquisa. 
Figura 1 - Framework da pesquisa

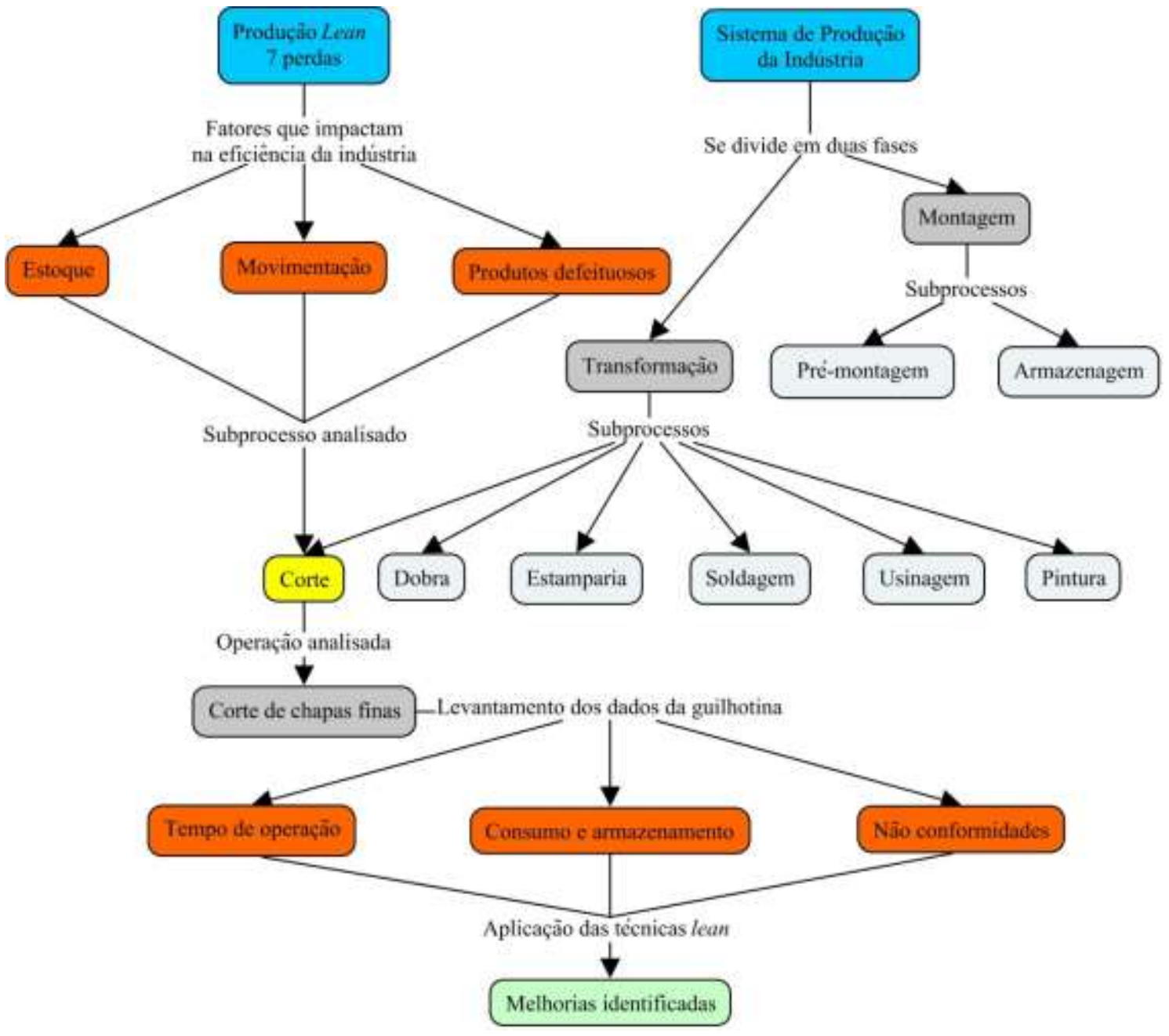

Fonte: Elaborado pelos autores

Também não há medidas e ações implantadas institucionalmente para o aproveitamento de retalhos das chapas. Assim como as portas, chapas possuem dimensões variadas. Normalmente cada chapa pode gerar duas portas e uma sobra, onde essa sobra poderia ser utilizada para a fabricação de outros itens. A ausência de controle de estoque de retalhos de chapas, com informações sobre tamanhos, tipos de chapa e quantidades, torna praticamente inviável aproveitar os segmentos remanescentes.

\subsection{Controle de Estoque de Matéria-Prima e Dimensionamento}

O armazenamento de retalhos é feito através do empilhamento das sobras de material, sem controle formal sobre a quantidade e tampouco sobre a localização desse material. Não existe a possibilidade de acessar chapas que estão armazenadas há muito tempo, pois ficam sob chapas 
colocadas mais recentemente. A busca por esses retalhos requereria um tempo muito elevado para a busca e manuseio dessas chapas. Não estando em condições ideais de armazenamento, ocorrem com frequência arranhões e amassados, inutilizando-as.

A gestão eficiente do estoque reduz significativamente gastos relativos a quantidade, movimentação e espaço necessário para a operacionalização de um processo. Na fabricação de portas, que se inicia na guilhotina, não há gestão de estoque. Não há quantidade e nem localização definidas para o armazenamento de matéria-prima. Além disso, inúmeras chapas estão danificadas devido às condições descritas.

\section{Aplicação das Práticas Lean \\ 4.1 Tempos de Operação}

A utilização do tempo da guilhotina responsável pelo corte de perfis de portas, foi obtido através de dez seções de medições com duração de aproximadamente uma hora, in loco, no período de três semanas, em horários e com operadores aleatórios. Foram tomados os tempos de setup, corte, carregamento de MP na máquina, organização de sequência de operação, ajustes, inspeção de item cortado, transporte de MP manual e tempo de espera. A partir desses dados, obteve-se o tempo em operação. O resultado obtido foi comparado com o monitoramento eletrônico e dessa forma, validado. Com base nesses dados de operação foi possível fazer uma aproximação da real capacidade de operação da máquina e projetar corretamente sua possibilidade de carga. A Tabela 1 apresenta a utilização média diária da distribuição das atividades analisadas pelo tempo total disponível da máquina.

Tabela 1 - Utilização média do tempo da máquina

\begin{tabular}{lllll}
\hline & \% total do tempo & tempo total & tempo médio da tarefa & d. padrão \\
\hline Setup $/ 1^{\circ}$ item & $31 \%$ & 185 & 3,68 & 1,96 \\
Espera & $20 \%$ & 126 & 5,31 & 5,64 \\
Cortar (x2) & $20 \%$ & 132 & 1,17 & 0,60 \\
Ajuste de guilhotina & $8 \%$ & 49 & 10,37 & 4,46 \\
Transportar chapa manualmente & $8 \%$ & 47 & 1,41 & 0,57 \\
Colocar pallets de chapas & $5 \%$ & 6 & 1,23 & 0,17 \\
Inspecionar item cortado & $5 \%$ & 38 & 1,11 & 0,32 \\
Organizar sequência & $4 \%$ & 24 & 2,49 & 0,75 \\
Total & $100,00 \%$ & 607 & & \\
\hline
\end{tabular}

Fonte: Elaborado pelos autores

O tempo médio para posicionamento e corte de chapa na guilhotina é de 35 segundos e o tempo médio gasto para o setup/Fabricação do $1^{\circ}$ item é de 3 minutos e 41 segundos. Não é possível separar 
as operações de setup e $1^{\circ}$ item fabricado, pois são realizados simultaneamente e são diretamente dependentes.

Projetou-se o tempo necessário, em condições ideais, para a fabricação de um plano diário médio - 20 elevadores, com média de 12 andares - considerando tempos de setup, posicionamento, corte e ajuste de máquina, além da adição de $15 \%$ de folga devido à variabilidade. A fabricação do plano consumiria $72,59 \%$ da carga disponível, restando ainda 27,41\% para outros painéis e itens urgentes. Com esses dados, se organizou o planejamento da produção diária já sequenciado, além de janelas de tempo para a fabricação de itens urgentes. Essas medidas impactaram na quantidade de setups, eliminação da necessidade de organizar a sequência pelo operador e redução da necessidade de transportar chapas manualmente.

\subsection{Consumo e Armazenamento}

O primeiro passo foi a identificação da utilização de MP pelo posto através do sistema da empresa, de modo a criar uma solução de armazenamento eficiente e justo às demandas da produção. Para o cálculo de dimensionamento, como não há informações diretas acerca do consumo de chapas na fabricação de portas e o estoque delas é controlado por peso, levantaram-se os seguintes dados: quantidade de portas por tipo de chapa no período; medidas de altura e largura de corte para fabricação de portas; quantidade de tipos de chapas utilizadas; dimensões e densidade das chapas $\left(\mathrm{Kg} / \mathrm{m}^{2}\right)$; quantidade de portas (por código) por chapa correspondente; e peso das portas. Os dados consolidados referentes ao ano fiscal (FY) 2015/2016 podem ser observados na Tabela 2.

Tabela 2 - Consumo de chapas finas para fabricação de portas FY15/16

\begin{tabular}{|c|c|c|c|c|c|c|c|c|c|c|c|}
\hline Chapa & $\begin{array}{l}\text { Área } \\
\left(\mathbf{m}^{2}\right)\end{array}$ & $\begin{array}{l}\text { Qtde. de } \\
\text { Pavimentos }\end{array}$ & $\begin{array}{l}\text { custo } \\
(\mathbf{R} \$ / \mathrm{kg})\end{array}$ & $\begin{array}{l}\text { Peso/ } \\
\text { M2 }\end{array}$ & $\begin{array}{l}\text { Quantidade de } \\
\text { chapas }\end{array}$ & $\begin{array}{l}\text { Área } \\
\text { necessária } \\
\left(\mathbf{m}^{2}\right)\end{array}$ & $\begin{array}{l}\text { Área } \\
\text { utilizada } \\
\left(\mathbf{m}^{2}\right)\end{array}$ & $\begin{array}{l}\text { Qtde. Anual } \\
\text { (Ton/ano) }\end{array}$ & $\begin{array}{l}\text { Qtde } \\
\text { Média } \\
\text { (Ton/mês) }\end{array}$ & $\begin{array}{l}\text { Qtde. } \\
\text { Pallets } \\
(\mathbf{1 , 8 T}) / \mathrm{Mês}\end{array}$ & $\begin{array}{l}\mathbf{A} \\
\mathbf{B} \\
\mathbf{C}\end{array}$ \\
\hline 009.141 & 2,417 & 10204 & 3,40 & 7,7 & 10911 & 22705,14 & 26369,70 & 203,05 & 16,92 & 9,40 & A \\
\hline 009.138 & 2,417 & 10419 & 3,21 & 7,7 & 10636 & 23284,15 & 25705,08 & 197,93 & 16,49 & 9,16 & A \\
\hline 001.092 & 2,417 & 9813 & 8,64 & 7,2 & 9813 & 21710,06 & 23716,06 & 170,76 & 14,23 & 7,91 & A \\
\hline 001.091 & 2,614 & 5323 & 10,51 & 7,2 & 5323 & 12884,41 & 13911,85 & 100,17 & 8,35 & 4,64 & B \\
\hline 009.142 & 2,573 & 3629 & 3,21 & 7,7 & 3970 & 8734,53 & 10214,02 & 78,65 & 6,55 & 3,64 & B \\
\hline 009.139 & 2,573 & 3460 & 4,00 & 7,7 & 3862 & 8611,60 & 9936,15 & 76,51 & 6,38 & 3,54 & B \\
\hline 001.040 & 2,517 & 2622 & 12,46 & 7,2 & 3001 & 6027,05 & 7555,02 & 54,40 & 4,53 & 2,52 & B \\
\hline 001.113 & 1,614 & 2036 & 14,36 & 7,2 & 4072 & 5776,68 & 6573,97 & 47,73 & 3,98 & 2,21 & B \\
\hline 001.114 & 1,667 & 1513 & 15,71 & 7,2 & 3026 & 4575,60 & 5044,77 & 36,63 & 3,05 & 1,70 & $\mathrm{C}$ \\
\hline 001.044 & 2,680 & 1536 & 13,01 & 7,2 & 1814,5 & 3855,71 & 4862,86 & 35,01 & 2,92 & 1,62 & $\mathrm{C}$ \\
\hline 001.112 & 1,818 & 891 & 14,82 & 7,2 & 1782 & 2973,73 & 3239,22 & 23,52 & 1,96 & 1,09 & $\mathrm{C}$ \\
\hline
\end{tabular}




\begin{tabular}{|c|c|c|c|c|c|c|c|c|c|c|c|}
\hline 009.172 & 2,573 & 935 & 3,92 & 7,7 & 935 & 2117,05 & 2405,57 & 18,52 & 1,54 & 0,86 & $\mathrm{C}$ \\
\hline 001.080 & 3,048 & 574 & 10,72 & 7,2 & 590 & 1484,28 & 1798,03 & 13,05 & 1,09 & 0,60 & $\mathrm{C}$ \\
\hline 001.093 & 2,537 & 625 & 7,12 & 7,2 & 625 & 1490,73 & 1585,50 & 11,42 & 0,95 & 0,53 & $\mathrm{C}$ \\
\hline 001.068 & 3,125 & 270 & 13,22 & 7,2 & 415 & 774,35 & 1296,88 & 9,34 & 0,78 & 0,43 & $\mathrm{C}$ \\
\hline 001.041 & 2,643 & 297 & 12,98 & 7,2 & 368 & 725,38 & 972,44 & 7,00 & 0,58 & 0,32 & $\mathrm{C}$ \\
\hline 009.140 & 3,000 & 80 & 3,41 & 7,7 & 160 & 226,37 & 480,00 & 3,70 & 0,31 & 0,17 & $\mathrm{C}$ \\
\hline 009.171 & 2,417 & 138 & 2,92 & 7,7 & 138 & 322,68 & 333,52 & 2,57 & 0,21 & 0,12 & $\mathrm{C}$ \\
\hline 009.143 & 3,000 & 40 & 3,79 & 7,7 & 80 & 123,87 & 240,00 & 1,85 & 0,15 & 0,09 & $\mathrm{C}$ \\
\hline 001.001 & 2,515 & 12 & 12,63 & 10,16 & 12 & 26,92 & 30,18 & 0,31 & 0,03 & 0,01 & $\mathrm{C}$ \\
\hline Total & 50,16 & 54417 & - & - & 61534 & 128430,31 & 146270,81 & 1092,08 & 91,01 & 50,56 & - \\
\hline
\end{tabular}

Fonte: Elaborado pelos autores

Observa-se que existe uma diferença superior a 140 toneladas entre o peso utilizado em chapas e o peso líquido das portas. Essa diferença se deve à geração de retalhos e sobras no processo de corte das chapas, que são absorvidas no gasto de fabricação de perfis de portas, elevando a ineficiência e o valor final. Se observa também que existe uma defasagem entre o peso por metro quadrado de chapa registrado no sistema e o que entra na fábrica de fato, além da variação entre pallets. Esses fatores chegam a gerar mais de $13 \%$ de inconsistência entre o estoque físico e o registrado no sistema. Com a quantidade de portas e seu peso correspondente, foi possível calcular o quanto foi utilizado de matériaprima durante o último ano fiscal, que é apresentado na Tabela 3.

Tabela 3 - Consumo médio mensal de chapas de aço (FY 15/16)

\begin{tabular}{lll}
\hline Chapa & Qtde. Anual (Ton./ano) & Qtde. Média (Ton./mês) \\
\hline 009.141 & 203,05 & 16,92 \\
009.138 & 197,93 & 16,49 \\
001.092 & 170,76 & 14,23 \\
001.091 & 100,17 & 8,35 \\
009.142 & 78,65 & 6,55 \\
009.139 & 76,51 & 6,38 \\
001.040 & 54,4 & 4,53 \\
001.113 & 47,73 & 3,98 \\
001.114 & 36,63 & 3,05 \\
001.044 & 35,01 & 2,92 \\
001.112 & 23,52 & 1,96 \\
009.172 & 18,52 & 1,54 \\
001.080 & 13,05 & 1,09 \\
001.093 & 11,42 & 0,95 \\
001.068 & 9,34 & 0,78 \\
001.041 & 7 & 0,58
\end{tabular}




$\begin{array}{lll}009.140 & 3,7 & 0,31 \\ 009.171 & 2,57 & 0,21 \\ 009.143 & 1,85 & 0,15 \\ 001.001 & 0,31 & 0,03\end{array}$

Fonte: Elaborado pelos autores

A partir dos volumes de utilização calculados, foi implementado no setor um magazine de chapas classificadas como A e B (ver Tabela 1), para reduzir as movimentações e necessidades de empilhadeiras para abastecer o setor. Esta implementação foi realizada com o objetivo de diminuir principalmente o tempo de espera por matéria-prima.

\subsection{Não Conformidades}

No relatório de não conformidades da empresa, a quantidade de produtos não conformes gerados diretamente (quando a própria máquina é a causadora) ou indiretamente (quando a não conformidade é ocasionada pelos setores seguintes, porém devido a algum erro do setor) é muito elevada. As principais causas de falhas, representam $75 \%$ do valor total e são respectivamente: produtos arranhados, produtos amassados e produtos fabricados a mais do que o necessário. A Tabela 4 apresenta a incidência de não conformidades ocasionadas no ano fiscal 2015/2016:

Tabela 4 - Quantidade e Valor de Não-Conformidades (FY 15/16)

\begin{tabular}{llll}
\hline Defeito & Qtde. & Valor & \% do total \\
\hline Arranhado & 455 & $\mathrm{R} \$ 47,925.44$ & $36 \%$ \\
Amassado & 326 & $\mathrm{R} \$ 30,515.01$ & $23 \%$ \\
Produção excedente & 875.99 & $\mathrm{R} \$ 20,433.85$ & $16 \%$ \\
\hline
\end{tabular}

Fonte: Elaborado pelos autores

Percebe-se que o defeito que apresenta maior quantidade foi a produção excedente além do que foi determinado pelas OFs. No entanto, a que representa maior valor e porcentagem do total são as peças arranhadas.

\section{Resultados}

Por meio dos tempos expressos na Tabela 1, observa-se que menos de 51\% do tempo é gasto em tarefas que agregam valor. A organização das chapas de forma adequada e otimizada para a utilização agiria sobre os $20 \%$ do tempo que é gasto em espera. A reorganização do método da disposição de chapas para o carregamento da máquina através do sequenciamento, atingiria os $8 \%$ de tempo em 
transporte e $1 \%$ em posicionamento. Além disso, é possível eliminar $4 \%$ do tempo gasto em organização de OFs com as medidas mencionadas. Assim, em condições ideais, seria possível otimizar mais de 30\% do tempo apenas com medidas de organização e sequenciamento.

Na Figura 2 estão os valores do uptime antes e após as melhorias realizadas no setor. Observa-se que houve uma melhora considerável nos pontos críticos abordados no processo de melhoria. O uptime da máquina, passou de $30,68 \%$ de média nos 10 meses antecedentes às mudanças, para 56\% nos 10 meses posteriores ao trabalho realizado.

Figura 2 - Uptime guilhotina comparando os 10 meses anteriores e posteriores a implantação (monitoramento eletrônico)

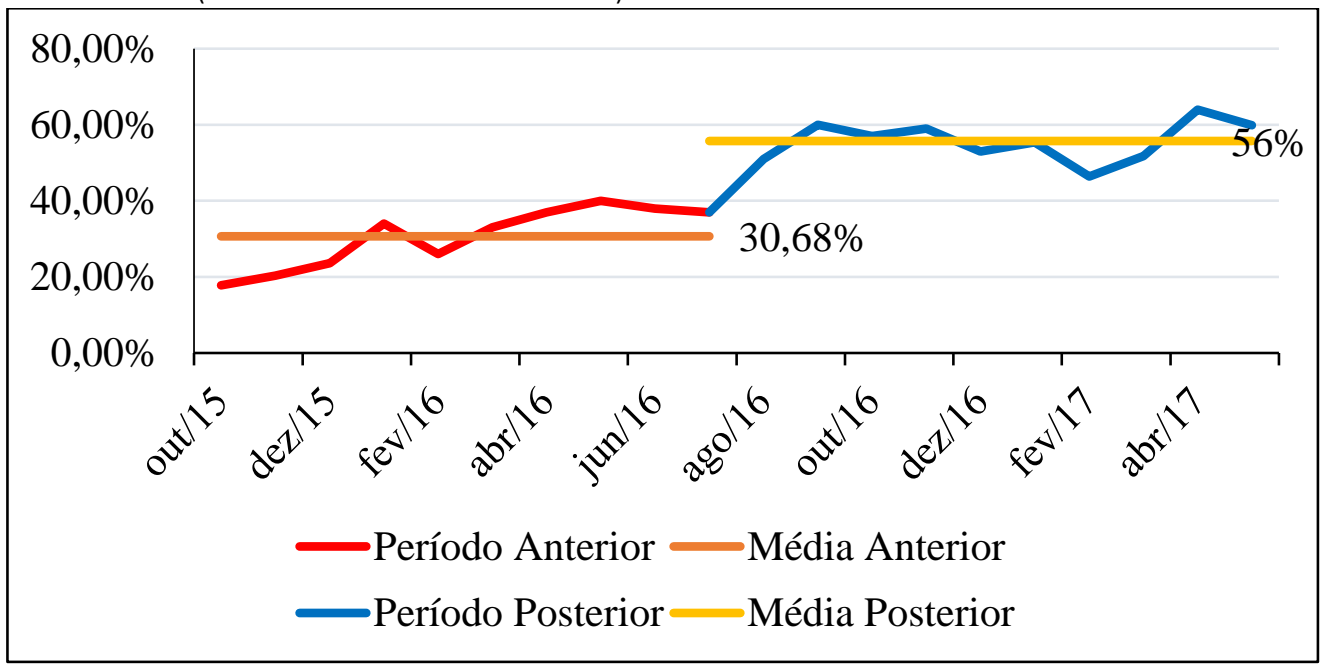

Fonte: Elaborado pelos autores

O principal resultado obtido, além de uma melhora significativa na organização do setor e eficiência, foi a redução dos gastos gerados por não conformidades. Na Tabela 5 é possível observar uma melhora significativa na quantidade de NC e seu impacto na redução de custos em relação ao período anterior às melhorias lean. 
Tabela 5 - Comparação (FY 15/16 - 16/17). Evidência das melhorias realizadas no setor

\begin{tabular}{lllll}
\hline Defeito & Qtde. & Valor & \% do total & Redução \\
\cline { 2 - 5 } Antes (FY 15/16) & & & & \\
Arranhado & 455 & $\mathrm{R} \$ 47,925.44$ & $36 \%$ & - \\
Amassado & 326 & $\mathrm{R} \$ 30,515.01$ & $23 \%$ & - \\
Produção excedente & 875 & $\mathrm{R} \$ 20,433.85$ & $16 \%$ & - \\
\hline Depois (FY 16/17) & & & & \\
Arranhado & 78 & $\mathrm{R} \$ 8,645.94$ & $22 \%$ & $\mathbf{8 3 \%}$ \\
Amassado & 73 & $\mathrm{R} \$ 7,379.87$ & $19 \%$ & $\mathbf{7 8 \%}$ \\
Produção excedente & 82 & $\mathrm{R} \$ 868.35$ & $2 \%$ & $\mathbf{9 1 \%}$ \\
\hline
\end{tabular}

Fonte: Elaborado pelos autores

Percebe-se que há uma melhor organização do setor com melhorias proporcionadas à operação. A movimentação dos pallets é realizada por empilhadeiras. Anteriormente, para ter acesso a uma chapa era necessário a remoção de todos os pallets que se encontravam na parte superior. Além do tempo excessivo de movimentação, este armazenamento acarretava em danos físicos às chapas. A Figura 3 apresenta a situação do estoque de matéria prima antes e depois das melhorias propostas e implementadas.

Figura 3 - Estoque de matéria-prima antes (a) e após (b) as melhorias implantadas

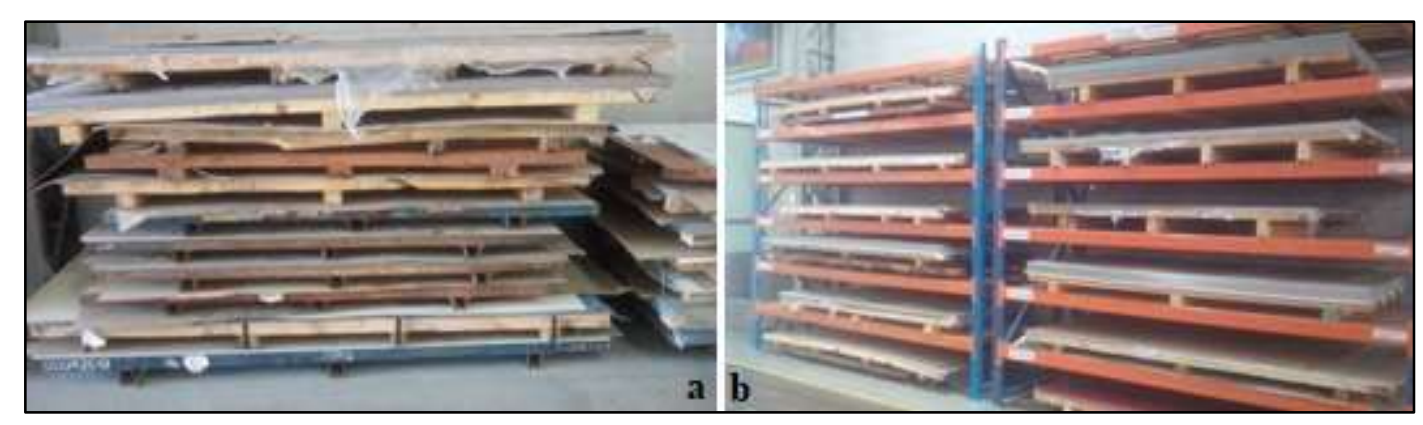

Fonte: Elaborado pelos autores

Observa-se a partir das mudanças realizadas melhorias significativas que foram proporcionadas na gestão visual dos estoques e suas condições a partir do armazenamento em estantes, classificados com os materiais identificados e ordenados conforme consumo apontado pela classificação $A B C$ apresentado na Tabela 2.

\section{Conclusões}

O trabalho demonstrou que soluções simples de melhoria, como a gestão de estoques para a máquina e a organização de ordens de fabricação para organizar a produção de modo geral, podem 
trazer impactos positivos significativos para na empresa analisada. Estas ações trouxeram uma redução de $69 \%$ no valor das perdas causadas por não conformidades. Além disso, foi possível identificar que a linha não está saturada e, portanto, modificações e ampliações resultariam em investimentos maiores em relação ao ganho obtido. Da mesma forma, aumento da quantidade de pessoas acarretaria em maior ociosidade e consequentemente uma menor produtividade.

A melhor alternativa encontrada para o aumento da produção e que se aproxima do tempo ideal de produção, refere-se à logística de entrega e recebimento de materiais. Aconselha-se que a empresa realize uma criação de intervalos de tempos (janelas) pré-definidos para a fabricação de itens urgentes, permitindo assim uma separação antecipada de chapas para os itens previstos, otimizando o uso da máquina através da redução do tempo de espera de material. Por fim, a pesquisa contribui para a lacuna de informações sobre o lean nos processos produtivos industriais, com ênfase para a temática da redução de custos e resíduos de matéria-prima.

A pesquisa apresentou como limitação a análise de um subprocesso de uma fábrica de elevadores localizada na região sul do Brasil. Desse modo, como propostas para trabalhos futuros, sugere-se: i) analisar outros processos produtivos da empresa; ii) realizar o mapeamento de fluxo de valor da empresa e identificar pontos de melhorias (Kaizen); e iii) analisar o impacto da criação de um modelo otimizado para o setor de corte.

\section{Referências}

Aherne, J., \& Whelton, J. (2010). Applying lean in healthcare : a collection of international case studies. CRC Press.

Alkhoraif, A., \& McLaughlin, P. (2018). Lean implementation within manufacturing SMEs in Saudi Arabia: Organizational culture aspects. Journal of King Saud University - Engineering Sciences, v. 30, n. 3, p. 232-242. https://doi.org/10.1016/j.jksues.2018.04.002

Bergek, A., Hekkert, M., Jacobsson, S., Markard, J., Sandén, B., \& Truffer, B. (2015). Technological innovation systems in contexts: Conceptualizing contextual structures and interaction dynamics. Environmental Innovation and Societal Transitions, 16, 51-64. https://doi.org/10.1016/j.eist.2015.07.003

Bortolotti, T., Boscari, S., \& Danese, P. (2015). Successful lean implementation: Organizational culture and soft lean practices. International Journal of Production Economics, 160, 182-201. https://doi.org/10.1016/J.IJPE.2014.10.013

Cançado, V. L., \& Santos, T. M. C. (2014). Reação à Mudança Organizacional: A Implantação do Lean Thinking na Empresa Beta. Revista Gestão \& Tecnologia, 14(1), 100-125. https://doi.org/10.20397/2177-6652/2014.v14i1.592

Coronel, D. A. (2014). Política industrial e desenvolvimento econômico : a reatualiação de um debate histórico, 34(134), 103-119.

Cortes, H. et al. (2016). Strategic Lean Management: Integration of operational Performance Indicators for strategic Lean management. IFAC-PapersOnLine, v. 49, n. 12, p. 65-70. https://doi.org/10.1016/j.ifacol.2016.07.551

Este, P. D., lammarino, S., Savona, M., \& Tunzelmann, N. Von. (2012). What hampers innovation ? Revealed barriers versus deterring barriers. Research Policy, 41(2), 482-488. 
https://doi.org/10.1016/j.respol.2011.09.008

Fliedner, G., \& Majeske, Ka. (2010). Sustainability: The New Lean Frontier. Production and Inventory Management Journal, 46(1), 6-13.

Giannini, R. (2007). Aplicação de ferramentas do pensamento enxuto na redução de perdas em operações de serviços, 121.

Gil, A. C. (2012). Como elaborar projetos de pesquisa. 5 ed. São Paulo: Atlas.

Hasibul, I. M., Gustav, B., \& Malin, T. (2018), Adoption of lean philosophy in car dismantling companies in Sweden-a case study. Procedia Manufacturing, v. 25, p. 620-627. https://doi.org/10.1016/j.promfg.2018.06.093

Hines, P., Holweg, M., \& Rich, N. (2004). Learning to evolve. International Journal of Operations \& Production Management, 24(10), 994-1011. https://doi.org/10.1108/01443570410558049

Ledón, R. A. et al. (2018). A meta-analytic study of the impact of Lean Production on business performance. International Journal of Production Economics, v. 200, p. 83-102. https://doi.org/10.1016/j.ijpe.2018.03.015

Lermen, F. H. et al. (2018). A framework for selecting lean practices in sustainable product development: The case study of a Brazilian agroindustry. Journal of Cleaner Production, v. 191, p. 261-272. https://doi.org/10.1016/j.jclepro.2018.04.185

Liker, J. K. (2004). The Toyota way : 14 management principles from the world's greatest manufacturer. McGraw-Hill.

Marodin, G. et al. (2018). Lean product development and lean manufacturing: Testing moderation effects. International Journal of Production Economics, v. 203, p. 301-310. https://doi.org/10.1016/j.ijpe.2018.07.009

Negri, F. D. D., \& Cavalcante, L. R. (2013). Análise dos dados da PINTEC 2011. Nota Técnica No 15, 9.

Ohno, T. (1988). Toyota production system : beyond large-scale production. Productivity Press.

Oliveira, C. (2009). Aplicação de técnicas de simulação em projetos de manufatura enxuta. Estudos Tecnológicos Em Engenharia, 4(3), 204-217. https://doi.org/10.4013/ete.20083.06

Palisaitiene, J. K., Sundin, E., \& Poksinska, B. (2018). Remanufacturing challenges and possible lean improvements. Journal of Cleaner Production, v. 172, p. 3225-3236. https://doi.org/10.1016/j.jclepro.2017.11.023

Pearce, A., Pons, D., \& Neitzert, T. (2018). Implementing lean-Outcomes from SME case studies. Operations Research Perspectives, v. 5, p. 94-104. https://doi.org/10.1016/j.orp.2018.02.002

Salgado, E. G., Mello, C. H. P., Silva, C. E. S. da, Oliveira, E. da S., \& Almeida, D. A. de. (2009). Análise da aplicação do mapeamento do fluxo de valor na identificação de desperdícios do processo de desenvolvimento de produtos. Gestão \& Produção, 16(3), 344-356. https://doi.org/10.1590/S0104-530X2009000300003

Sharma, S., \& Gandhi, P. J. (2017). Scope and Impact of Implementing Lean Principles \& Practices in Shipbuilding. Procedia Engineering, v. 194, p. 232-240. https://doi.org/10.1016/j.proeng.2017.08.140

Shah, R., \& Ward, P. T. (2003). Lean manufacturing: Context, practice bundles, and performance. Journal of Operations Management, 21(2), 129-149. https://doi.org/10.1016/S02726963(02)00108-0

Shingō, S., \& Dillon, A. P. (1989). A study of the Toyota production system from an industrial engineering viewpoint. Productivity Press.

Slack, N., Chambers, S., Johnston, R., Oliveira, M. T. C. de, \& Alher, F. (2002). Administração da produção. Atlas.

Soliman, M., \& Saurin, T. A. (2017). Lean production in complex socio-technical systems: A systematic literature review. Journal of Manufacturing Systems, v. 45, p. 135-148.

https://doi.org/10.1016/j.jmsy.2017.09.002

Utterback, J. M., \& Abernathy, W. J. (1975). A dynamic model of process and product innovation. Omega, 3(6), 639-656. https://doi.org/10.1016/0305-0483(75)90068-7

Womack, J. P., \& Jones, D. T. (2004). A mentalidade enxuta nas empresas : elimine o desperdício e crie riqueza. Campus. 
Yin, R. K. (2001). Estudo de caso. Planejamento e métodos. 2a ed., Porto Alegre/RS: Bookmam.

Recebido em: 14 ago. 2018 / Aprovado em: 24 jan. 2019

\section{Para referenciar este texto}

Vecchia, F. A. D., Volquind, R., Fernandes, E. da S., Silveira, F. da, Santos, B. M. dos, \& Amaral, F. G. (2020). Práticas lean nos processos produtivos industriais: ações para a redução de custos e resíduos de matéria-prima. Exacta, 18(1), 1-15. https://doi.org/10.5585/Exacta.v18n1.10281. 